\title{
Thermal Behavior of the Compactified 3-D Gross-Neveu Model
}

\author{
A. P. C. Malbouisson, \\ Centro Brasileiro de pesquisas Físicas/MCT, 22290-180, Rio de Janeiro, RJ, Brazil \\ F. C. Khanna, \\ Theoretical Physics Institute, University of Alberta, Edmonton, AB T6G 2J1, Canada
}

J. M. C. Malbouisson,

Intituto de Física, Universidade Federal da Bahia, 40210-340, Salvador, BA, Brazil

and A. E. Santana

Instituto de Física, Universidade de Brasília, 70910-900, Brasília, DF, Brazil

Received on 10 August, 2006

\begin{abstract}
We consider the $N$-component tri-dimensional massive Gross-Neveu model at finite temperature and with compactified spatial coordinates. We study the behavior of the renormalized large- $N$ effective coupling constant, investigating its dependence on the compactification length and the temperature. We show that spatial confinement exists for the model at $T=0$, which is destroyed by raising the temperature.
\end{abstract}

Keywords: Four-fermions interactions; Spatial confinement; Thermal deconfinement

\section{INTRODUCTION}

In the most commonly accepted scenario for the early evolution of the universe, the hadronic matter emerges in a confining phase transition where the quark-gluon plasma condenses into material "droplets," the hadrons, as the universe cooled down. In the context of the standard model, the quantum chromodynamics (QCD) should then be able to predict color and spatial confinement, since hadrons are colorless and their constituents, quarks and gluons, are confined in a region having a linear size of order $1 \mathrm{fm}$ (fossil quarks have never been observed). Also, quarks are asymptotically free, the interaction between them being very weak, at very high energies as probed by deep inelastic scattering processes. QCD should also account for the fact that the hadronization transition occurred in the early universe at a finite temperature, estimated of the order of $200 \mathrm{MeV}$.

Nevertheless, although lattice calculations have given clear indications, up to present day, no fully analytical treatment with the standard model has been constructed capable of taking into account simultaneously all these different aspects of hadronic matter. This is mainly due to the intricate field theoretical structure of non-Abelian QCD and, for this reason, effective theories and phenomenological approaches have been developed over the last decades. The simplest effective theory is the celebrated Gross-Neveu (GN) model [1], which considers direct four-fermions interaction.

In this spirit, we shall investigate the tri-dimensional GrossNeveu model, in the large- $N$ limit, at finite temperature and with one or two compactified spatial dimensions. Our aim is to study analytically the simultaneous occurrence of spatial confinement and asymptotic freedom, and investigate the thermal deconfining transition. For that we will determine the dependence of the renormalized effective coupling on the compactification length and the temperature.

In this work, we first review our results for the 3-D GN model with one compactified spatial coordinate, both at zero and finite temperature, presented earlier $[2,3]$. Then, we extend the analysis to the case where all spatial dimensions are compactified.

\section{COMPACTIFIED GROSS-NEVEU MODEL}

Our starting point is the Wick-ordered massive GrossNeveu Lagrangian in a $D$-dimensional Euclidean space,

$$
\mathcal{L}=: \bar{\psi}(x)(i \not \nabla+m) \psi(x):+\frac{u}{2}(: \bar{\psi}(x) \psi(x):)^{2},
$$

where $m$ is the mass, $u$ is the coupling constant, $x$ is a point of $\mathbf{R}^{D}$ and the $\gamma$ 's are Dirac matrices. The quantity $\psi(x)$ is a spin $\frac{1}{2}$ field having $N$ (flavor) components, $\psi^{a}(x), a=1,2, \ldots, N$. Summation over flavor and spin indexes is understood. Here we consider the large- $N$ limit $(N \rightarrow \infty)$, which permits considerable simplification. We use natural units, $\hbar=c=k_{B}=1$.

The compactification is engendered via a generalized Matsubara prescription, which corresponds to considering the system in a topology $R^{D-d} \times S^{1_{1}} \times \cdots S^{1_{d}}$, thus treating spatial and imaginary-time compactifications on the same footing. To describe the model with $d(\leq D)$ compactified Euclidean coordinates, that is with $x_{i}$ restricted to segments of length $L_{i}(i=1,2, \ldots . d)$ and the field $\psi(x)$ satisfying anti-periodic (bag model) boundary conditions, the Feynman rules should be modified following the Matsubara replacements

$$
\int \frac{d k_{i}}{2 \pi} \rightarrow \frac{1}{L_{i}} \sum_{n_{i}=-\infty}^{+\infty}, \quad k_{i} \rightarrow \frac{2 \pi\left(n_{i}+\frac{1}{2}\right)}{L_{i}}
$$

Then the $L_{i}$-dependent four-point function at leading order in $1 / N$ and at zero external momenta, from which we will define the effective coupling constant between the fermions, 
has the formal expression

$$
\Gamma_{D d}^{(4)}\left(0 ;\left\{L_{i}\right\}, u\right)=\frac{u}{1+N u \Sigma_{D d}\left(\left\{L_{i}\right\}\right)},
$$

where $L_{i}$-dependent Feynman one-loop (bubble) diagram is given by

$$
\begin{aligned}
\Sigma_{D d}\left(\left\{L_{i}\right\}\right)= & \frac{1}{L_{1} \cdots L_{d}} \sum_{\left\{n_{i}\right\}=-\infty}^{\infty} \\
& \times \int \frac{d^{D-d} q}{(2 \pi)^{D-d}}\left[\frac{m^{2}-\mathbf{q}^{2}-\sum_{i=1}^{d} v_{i}^{2}}{\left(\mathbf{q}^{2}+\sum_{i=1}^{d} v_{i}^{2}+m^{2}\right)^{2}}\right]
\end{aligned}
$$

and $v_{i}=2 \pi\left(n_{i}+\frac{1}{2}\right) / L_{i}$.

In order to simplify the use of regularization techniques, we introduce the following dimensionless quantities: $q_{j}^{\prime}=$ $q_{j} / 2 \pi m(j=d+1, \ldots, D)$ and $b_{i}=\left(m L_{i}\right)^{-2}(i=1,2, \ldots, d)$. To proceed, we extend the method developed in Refs. $[2,3]$ to calculate the one-loop subdiagram, accounting for the compactification of $d(\leq D)$ Cartesian coordinates. We use a modified minimal subtraction scheme, employing concurrently dimensional and analytical regularizations, where the counterterms are poles of the Epstein-Hurwitz zeta-functions [2]. The calculations go through the following steps.

First, using dimensional regularization techniques to perform the integral over $\mathbf{q}^{\prime}$, Eq. (4) becomes

$$
\begin{aligned}
\Sigma_{D d}\left(\left\{b_{i}\right\}\right)= & \left.\Sigma_{D d}\left(s ;\left\{b_{i}\right\}\right)\right|_{s=2} \\
= & \frac{m^{D-2}}{(2 \pi)^{2}} \sqrt{b_{1} \cdots b_{d}} \\
& \times\left[\frac{1}{2 \pi^{2}} U_{D d}\left(s ;\left\{b_{i}\right\}\right)-U_{D}\left(s-1 ;\left\{b_{i}\right\}\right)\right]_{s=2},
\end{aligned}
$$

with

$$
\begin{aligned}
U_{D d}\left(\mu ;\left\{b_{i}\right\}\right)= & \pi^{\frac{(D-d)}{2}} \frac{\Gamma(v)}{\Gamma(\mu)} \\
& \times \sum_{\left\{n_{i}\right\}=-\infty}^{\infty}\left[\sum_{j=1}^{d} b_{j}\left(n_{j}+\frac{1}{2}\right)^{2}+(2 \pi)^{-2}\right]^{-v},
\end{aligned}
$$

where $\mathrm{v}=\mu-\frac{D-d}{2}$.

Secondly, transforming the summations over half-integers into sums over integers, Eq. (6) can be written as

$$
\begin{aligned}
U_{D d}\left(\mu ;\left\{b_{i}\right\}\right)= & \pi^{\frac{(D-d)}{2}} \frac{\Gamma(v)}{\Gamma(\mu)} 4^{v}\left[Z_{d}^{h^{2}}\left(v, b_{1}, \ldots, b_{d}\right)\right. \\
& -\sum_{i=1}^{d} Z_{d}^{h^{2}}\left(v, \ldots, 4 b_{i}, \ldots\right) \\
& +\sum_{i<j=1}^{d} Z_{d}^{h^{2}}\left(v, \ldots, 4 b_{i}, \ldots, 4 b_{j}, \ldots\right) \\
& \left.-\cdots+(-1)^{d} Z_{d}^{h^{2}}\left(v, 4 b_{1}, \ldots, 4 b_{d}\right)\right],
\end{aligned}
$$

where $h=\pi^{-1}$ and

$$
Z_{d}^{h^{2}}\left(v,\left\{a_{i}\right\}\right)=\sum_{\left\{n_{i}\right\}=-\infty}^{\infty}\left[\sum_{j=1}^{d} a_{j} n_{j}^{2}+h^{2}\right]^{-v}
$$

is the multiple-variable ( $d$-dimensional) Epstein-Hurwitz zeta-function. The function $Z_{d}^{h^{2}}\left(\mathrm{v},\left\{a_{i}\right\}\right)$ can be analytically extended to the whole complex $v$-plane [4], through a generalization of the procedure presented in Refs. [5, 6]; one finds

$$
\begin{aligned}
Z_{d}^{h^{2}}\left(\mathrm{v},\left\{a_{i}\right\}\right)= & \frac{\pi^{d / 2}}{\sqrt{a_{1} \cdots a_{d}} \Gamma(\mathrm{v})}\left[\frac{1}{h^{2(v-d)}} \Gamma\left(\mathrm{v}-\frac{d}{2}\right)\right. \\
& +4 \sum_{i=1}^{d} \sum_{n_{i}=1}^{\infty}\left(\frac{\pi n_{i}}{h \sqrt{a_{i}}}\right)^{v-\frac{d}{2}} K_{\mathrm{v}-\frac{d}{2}}\left(\frac{2 \pi h n_{i}}{\sqrt{a_{i}}}\right) \\
& +8 \sum_{i<j=1}^{d} \sum_{n_{i}, n_{j}=1}^{\infty}\left(\frac{\pi}{h} \sqrt{\frac{n_{1}^{2}}{a_{1}}+\frac{n_{2}^{2}}{a_{2}}}\right)^{v-\frac{d}{2}} \\
& \times K_{v-\frac{d}{2}}\left(2 \pi h \sqrt{\frac{n_{1}^{2}}{a_{1}}+\frac{n_{2}^{2}}{a_{2}}}\right)+\cdots \\
& +2^{d+1} \sum_{\left\{n_{i}\right\}=1}^{\infty}\left(\frac{\pi}{h} \sqrt{\frac{n_{1}^{2}}{a_{1}}+\cdots+\frac{n_{d}^{2}}{a_{d}}}\right)^{v-\frac{d}{2}} \\
& \left.\times K_{v-\frac{d}{2}}\left(2 \pi h \sqrt{\frac{n_{1}^{2}}{a_{1}}+\cdots+\frac{n_{d}^{2}}{a_{d}}}\right)\right]
\end{aligned}
$$

where $K_{v}(x)$ is the Bessel function of the third kind.

Thirdly, we notice that the first term in the square bracket of Eq. (9) leads to the following, $b_{i}$-independent, contribution to $\Sigma_{D d}\left(s ;\left\{b_{i}\right\}\right)$ :

$$
\begin{aligned}
\Sigma_{D d}^{\text {polar }}(s)= & \frac{m^{D-2} \pi^{\frac{D}{2}}}{(2 \pi)^{D-2(s-2)} \Gamma(s)} \\
& \times\left[2 \Gamma\left(s-\frac{D}{2}\right)-(s-1) \Gamma\left(s-1-\frac{D}{2}\right)\right] .
\end{aligned}
$$

When we take $s=2$, for even dimensions $D \geq 2$, this term diverges due to the poles of the $\Gamma$-functions; therefore, it should be subtracted in order to obtain a finite, renormalized, $\Sigma$. For the sake of uniformity, this term should also be suppressed for odd dimensions $D$, when no poles of the $\Gamma$-functions are present; in such cases, we perform a finite renormalization. The renormalized one-loop (bubble) diagram, which arises from the regular part of the analytical continuation of the Epstein-Hurwitz zeta-function, is then given by

$$
\Sigma_{D d}^{R}\left(\left\{b_{i}\right\}\right)=\left[\Sigma_{D d}\left(s ;\left\{b_{i}\right\}\right)-\Sigma_{D d}^{\mathrm{polar}}(s)\right]_{s=2} .
$$

Inserting $\Sigma_{D d}^{R}\left(\left\{b_{i}\right\}\right)$ into Eq. (3) and taking the limit $N \rightarrow$ $\infty$, with $N u=\lambda$ fixed as usual, we find the large- $N$ effective 
( $\left\{b_{i}\right\}$-dependent) renormalized coupling constant, for $d(\leq D)$ compactified dimensions, as

$$
\begin{aligned}
g_{D d}\left(\left\{b_{i}\right\}, \lambda\right) & =\lim _{N \rightarrow \infty}\left[N \Gamma_{D d}^{(4) R}\left(0,\left\{b_{i}\right\}, u\right)\right] \\
& =\frac{\lambda}{1+\lambda \Sigma_{D d}^{R}\left(\left\{b_{i}\right\}\right)} .
\end{aligned}
$$

This is the basic result for subsequent analysis. But, instead of pursuing the problem for general dimension $D$, we shall restrict our discussion to the $D=3$ case considering, initially, the case where only one spatial dimension is compactified.

\section{THE 3-D GROSS-NEVEU MODEL WITH ONE COMPACTIFIED SPATIAL DIMENSION}

Taking $D=3$ and $d=1$ in the preceding formulas, and fixing $b_{1}=L^{-2}$ (thus measuring $L$ in units of $m^{-1}$, as it will be done from now on), we find

$$
\begin{aligned}
\Sigma_{31}^{R}(L)= & \frac{m}{\sqrt{2} \pi^{\frac{3}{2}}}\left[2 \sum_{n=1}^{\infty}\left(\sqrt{2 L n}-\frac{1}{\sqrt{2 L n}}\right) K_{\frac{1}{2}}(2 L n)\right. \\
& \left.-\sum_{n=1}^{\infty}\left(\sqrt{L n}-\frac{1}{\sqrt{L n}}\right) K_{\frac{1}{2}}(L n)\right]
\end{aligned}
$$

Using the expression for the Bessel function of order $\frac{1}{2}$,

$$
K_{ \pm \frac{1}{2}}(z)=\sqrt{\pi} \frac{\exp (-z)}{\sqrt{2 z}}
$$

and performing the resulting geometric series, Eq. (13) becomes

$$
\Sigma_{31}^{R}(L)=\frac{m}{2 \pi}\left[\frac{1}{L} \log \left(1+e^{-L}\right)-\frac{1}{1+e^{L}}\right] .
$$

The behavior of $\Sigma_{31}^{R}$ as a function of $L$ is illustrated in Fig. 1 . We find from Eq. (15) that $\Sigma_{31}^{R}(L)$ diverges $(\rightarrow+\infty)$ when $L \rightarrow$ 0 and tends to 0 , through negative values, as $L \rightarrow \infty$. Numerically, we find that $\Sigma_{31}^{R}(L)$ vanishes for $L=L_{\min } \simeq 1.14 \mathrm{~m}^{-1}$, being negative for $L>L_{\min }^{(1)}$; it also assumes a minimum (negative) value, $\Sigma_{31}^{R \min } \simeq-0.0091 \mathrm{~m}$, for $L=L_{\max } \simeq 1.84 \mathrm{~m}^{-1}$. This dependence of $\Sigma_{31}^{R}$ on $L$, in particular the fact that $\Sigma_{31}^{R}(L)$ is negative for $L>L_{\min }$, has remarkable influence on the behavior of the renormalized effective coupling constant.

In the present case, Eq. (12) becomes

$$
g_{31}(L, \lambda)=\frac{\lambda}{1+\lambda \Sigma_{31}^{R}(L)} .
$$

We first note that, due to the divergence of $\Sigma_{31}^{R}, g_{21}(L, \lambda)$ approaches 0 as $L \rightarrow 0$, independently of the value of $\lambda$; therefore, the system presents a kind of asymptotic-freedom behavior for short distances. On the other hand, starting from a low value of $L$ (within this region of asymptotic freedom) and increasing the size of the system, $g_{31}$ will present a divergence at

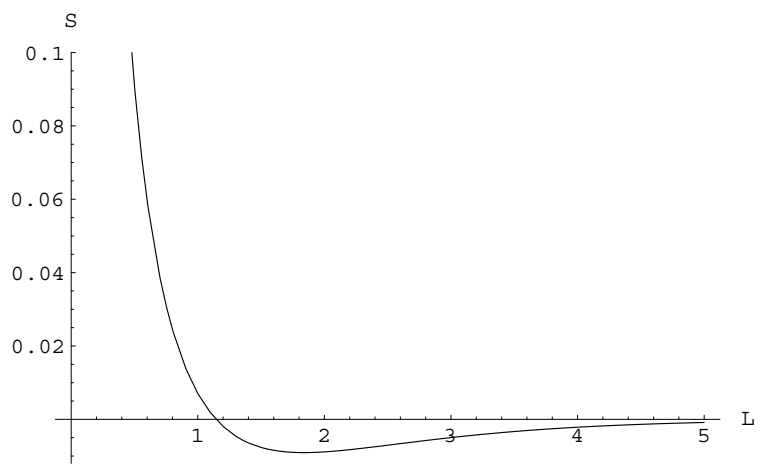

FIG. 1: Plot of $S=\Sigma_{31}^{R}(L) / m$ as a function of $L$ (in units of $m^{-1}$ ).

a finite value of $L\left(L_{c}\right)$, if the value of the fixed coupling con$\operatorname{stant}(\lambda)$ is high enough. In fact, this will happen for all values of $\lambda$ above the "critical value" $\lambda_{c}=\left(-\Sigma_{31}^{R \min }\right)^{-1} \simeq 110.0 \mathrm{~m}^{-1}$. The behavior of the effective coupling as a function of $L$ is illustrated in Fig. 2 for some values of the fixed coupling constant $\lambda$. We interpret this result by stating that, in the strongcoupling regime $\left(\lambda \geq \lambda_{c}\right)$ the system gets spatially confined in a segment of length $L_{c}(\lambda)$. We find also that

$$
L_{c}(\lambda) \in\left(L_{\min }, L_{\max }\right],
$$

with $L_{\max }$ corresponding to the confining length when $\lambda=\lambda_{c}$ while $L_{\min }$ is associated with $\lambda \rightarrow \infty$. The value of $L_{c}(\lambda)$, for an arbitrary value of $\lambda>\lambda_{c}$, can be found by searching for the smallest solution of the equation $1+\lambda \Sigma_{31}^{R}(L)=0$.

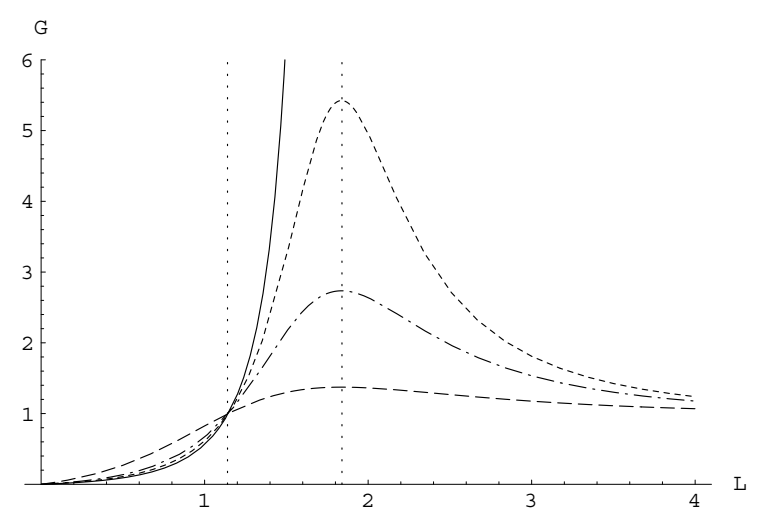

FIG. 2: Plots of the relative effective coupling constant, $G=$ $g_{31}(L, \lambda) / \lambda$, as a function of $L$ (in units of $m^{-1}$ ) for some values of $\lambda$ : $90 \mathrm{~m}^{-1}$ (dotted line), $70 \mathrm{~m}^{-1}$ (dotted-dashed line), $30 \mathrm{~m}^{-1}$ (dashed line) and $110 \mathrm{~m}^{-1}$ (full line). The vertical lines, passing by $L_{\min } \simeq 1.14$ and $L_{\max } \simeq 1.84$, are plotted as a visual guide.

We now consider the GN model, with one compactified spatial dimension, at finite temperature. To do so, we have to compactify the imaginary (Euclidean) time in a length $\beta=$ $1 / T$, where $T$ represents the temperature. Taking $b_{1}=L^{-2}$ 
and $b_{2}=\beta^{-2}$ in the formulas of Sec. II, we obtain

$$
\begin{aligned}
\frac{\sum_{32}^{R}(L, \beta)}{m}= & \frac{1}{2 \pi}\left[\frac{1}{L} \log \left(1+e^{-L}\right)+\frac{1}{\beta} \log \left(1+e^{-\beta}\right)\right] \\
& -\frac{1}{2 \pi}\left[\frac{1}{1+e^{L}}+\frac{1}{1+e^{\beta}}\right] \\
& +\frac{1}{\pi}\left[G_{2}(L, \beta)-2 G_{2}(L, 2 \beta)\right. \\
& \left.-2 G_{2}(2 L, \beta)+4 G_{2}(2 L, 2 \beta)\right]
\end{aligned}
$$

where the function $G_{2}(x, y)$ is defined by

$$
G_{2}(x, y)=\sum_{n, l=1}^{\infty} \exp \left(-\sqrt{x^{2} n^{2}+y^{2} l^{2}}\right)\left[1-\frac{1}{\sqrt{x^{2} n^{2}+y^{2} l^{2}}}\right] .
$$

Notice that the numerical computation of $\Sigma_{32}^{R}(L, \beta)$ is greatly facilitated by the fact that the double series defining the function $G_{2}(y, z)$ is rapidly convergent.

As a first remark, observe that $\Sigma_{32}^{R}(L, \beta)$ reduces to the expression (15) for the $T=0$ case, if we take $\beta \rightarrow \infty$. On the other hand, $\Sigma_{32}^{R}(L, \beta) \rightarrow \infty$ when $\beta \rightarrow 0$. This implies that, independently of the value of $\lambda$, the effective renormalized coupling constant,

$$
g_{32}(L, \beta, \lambda)=\frac{\lambda}{1+\lambda \Sigma_{32}^{R}(L, \beta)},
$$

vanishes when $\beta \rightarrow 0$ and, so, the system gets asymptotically free at very high temperatures, as expected. Therefore, if we start with the confined system $\left(\lambda \geq \lambda_{c}\right)$ at $T=0$ and raise the temperature, at some value $T=T_{d}(\lambda)$, the divergence of $g_{32}(L, \beta, \lambda)$ is suppressed, the system becoming unconfined.

For a fixed value of $\lambda\left(\geq \lambda_{c}\right)$, the determination of the deconfining temperature, $T_{d}(\bar{\lambda})$, can be implemented by examining of the minimum value of $g_{32}^{-1}(L, \beta, \lambda)$ for various values of $\beta$; the value of $\beta, \beta_{d}(\lambda)$, for which the minimum vanishes gives the deconfining temperature. In Fig. 3 , we plot $g_{32}^{-1}(L, \beta, 120)$ as a function of $L$, for some values of $\beta$, to illustrate the process. In this example with $\lambda=120 \mathrm{~m}^{-1}$, the minimum of $g_{32}^{-1}$ vanishes for $\beta=\beta_{d} \simeq 1.56 \mathrm{~m}^{-1}$ and is positive for $\beta>\beta_{d}$; thus, the deconfining temperature is $T_{d}=\beta_{d}^{-1} \simeq 0.64 m$ when $\lambda=120 m^{-1}$. For an arbitrary value of $\lambda\left(\geq \lambda_{c}\right)$, we have

$$
T_{d}(\lambda) \in\left[T_{d}^{\min }, T_{d}^{\max }\right)
$$

where $T_{d}^{\mathrm{min}} \simeq 0.6 \mathrm{~m}$ is the deconfining temperature when $\lambda=$ $\lambda_{c}$ and $T_{d}^{\max } \simeq 0.87 \mathrm{~m}$ is the upper bound, which would be only reached when $\lambda \rightarrow \infty$.

\section{THE 3-D GROSS-NEVEU MODEL WITH BOTH SPATIAL DIMENSIONS COMPACTIFIED}

We now consider the 3-dimensional GN model at $T=0$, where both spatial coordinates $x_{1}$ and $x_{2}$ have been compactified. Naturally, the expression for $\Sigma_{32}^{R}\left(L_{1}, L_{2}\right)$ can be obtained

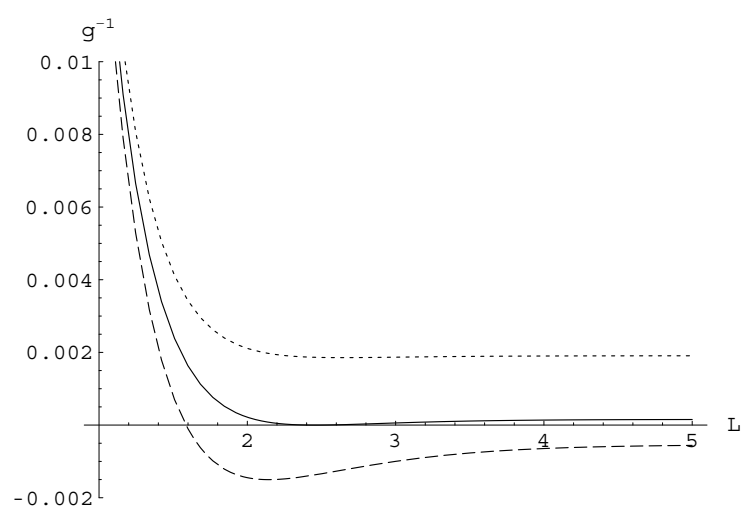

FIG. 3: Inverse of the effective coupling constant $g_{32}^{-1}$, with $\lambda=$ $120 \mathrm{~m}^{-1}$ fixed, as a function of $L$ (in units of $\mathrm{m}^{-1}$ ), for some values of $\beta$ (in units of $m^{-1}$ ): 2.0, 1.56 and 1.4 (dashed, full and dotted lines, respectively).

from Eq. (18) replacing $L$ and $\beta$ by $L_{1}$ and $L_{2}$. But, instead of considering the general situation, we shall restrict our analysis to the case where both compactification lengths $L_{1}$ and $L_{2}$ are equal to $L$. We then obtain

$$
\begin{aligned}
\frac{\Sigma_{32}^{R}(L)}{m}= & \frac{1}{\pi}\left[\frac{1}{L} \log \left(1+e^{-L}\right)-\frac{1}{1+e^{L}}\right] \\
& +\frac{1}{\pi}\left[G_{2}(L, L)-4 G_{2}(L, 2 L)+4 G_{2}(2 L, 2 L)\right],
\end{aligned}
$$

with the function $G_{2}(x, y)$ given by Eq. (19).

The function $\Sigma_{32}^{R}(L)$ has a behavior similar to that of $\Sigma_{31}^{R}(L)$, but the numbers are distinct: it vanishes at $L=L_{\min } \simeq$ $1.3 \mathrm{~m}^{-1}$ and assumes the minimum value, $\Sigma_{32}^{R \min } \simeq-0.0099 \mathrm{~m}$, for $L=L_{\max } \simeq 2.1 m^{-1}$. Also, $\Sigma_{32}^{R}(L)$ diverges when $L \rightarrow 0$ and tends to 0 , as $L \rightarrow \infty$. The plot of $\Sigma_{32}^{R}(L) / m$ is shown in Fig. 4.

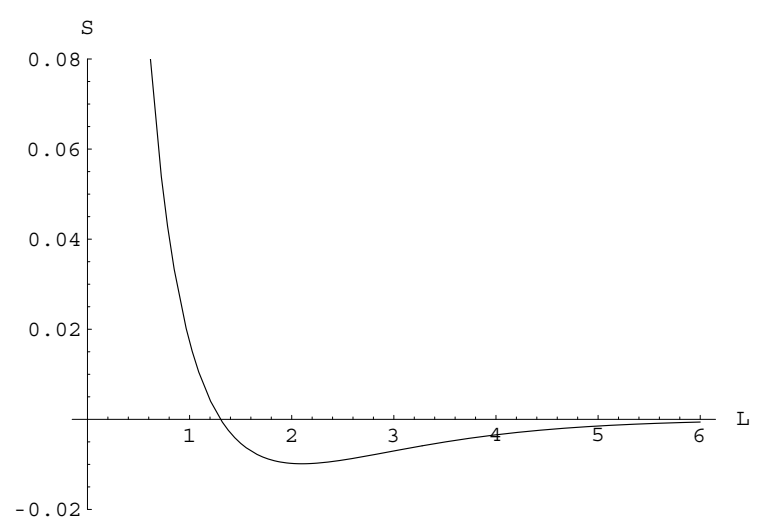

FIG. 4: Plot of $S=\Sigma_{32}^{R}(L) / m$ as a function of $L$ (in units of $m^{-1}$ ).

Thus, in the present case, the effective renormalized cou- 
pling constant,

$$
g_{32}(L, \lambda)=\frac{\lambda}{1+\lambda \Sigma_{32}^{R}(L)},
$$

maintains the same characteristics as before; it vanishes as $L \rightarrow 0$, irrespective to the value of $\lambda$, and for $\lambda \geq \lambda_{c}=$ $\left(-\Sigma_{32}^{R \min }\right)^{-1} \simeq 101.0 \mathrm{~m}^{-1}$, it presents a divergence at a finite value of $L, L_{c}(\lambda) \in\left(L_{\min }, L_{\max }\right]$. Therefore, in the fully compactified 3-D model, we still have a behavior that is quite similar to the asymptotic freedom for small distances and spatial confinement in the strong-coupling regime, $\lambda \geq \lambda_{c}$. Now, it remains to investigate the effect of finite temperature on the effective renormalized coupling constant.

To consider temperature effects, we have to compactifiy the imaginary time in a length $\beta=1 / T$. In this case, taking $L_{1}=L_{2}=L$ and $L_{3}=\beta$ in the formulas of Sec. II, we find the following expression for the $L-\beta$ dependent renormalized bubble diagram:

$$
\begin{aligned}
\frac{\sum_{33}^{R}(L, \beta)}{m}= & \frac{1}{2 \pi}\left[\frac{2}{L} \log \left(1+e^{-L}\right)+\frac{1}{\beta} \log \left(1+e^{-\beta}\right)\right] \\
& -\frac{1}{2 \pi}\left[\frac{2}{1+e^{L}}+\frac{1}{1+e^{\beta}}\right] \\
& +\frac{1}{\pi}\left[G_{2}(L, L)+2 G_{2}(L, \beta)-4 G_{2}(L, 2 L)\right. \\
& -4 G_{2}(L, 2 \beta)-4 G_{2}(2 L, \beta)+4 G_{2}(2 L, 2 L) \\
& +8 G_{2}(2 L, 2 \beta)-2 G_{3}(L, L, \beta)+4 G_{3}(L, L, 2 \beta) \\
& +8 G_{3}(2 L, L, \beta)-8 G_{3}(2 L, 2 L, \beta) \\
& \left.-16 G_{3}(2 L, L, 2 \beta)+16 G_{3}(2 L, 2 L, 2 \beta)\right],(24)
\end{aligned}
$$

where $G_{2}(x, y)$ is given by Eq. (19) and the function $G_{3}(x, y, z)$ is defined by

$$
\begin{aligned}
G_{3}(x, y, z)= & \sum_{n, l, r=1}^{\infty} \exp \left(-\sqrt{x^{2} n^{2}+y^{2} l^{2}+z^{2} r^{2}}\right) \\
& \times\left[1-\frac{1}{\sqrt{x^{2} n^{2}+y^{2} l^{2}+z^{2} r^{2}}}\right] .
\end{aligned}
$$

Note that, making $\beta \rightarrow \infty$, Eq. (24) reduces to $\Sigma_{32}^{R}(L)$, given by Eq. (22).

As before, the increase of the temperature destroys the spatial confinement that exists for $\lambda \geq \lambda_{c}$ at $T=0$. We can determine the deconfining temperature by searching for the value of $\beta(\lambda)$ for which the minimum of the inverse of the effective renormalized coupling constant, $g_{33}^{-1}(L, \beta, \lambda)=$ $\left(1+\lambda \Sigma_{33}^{R}(L, \beta)\right) / \lambda$, vanishes. For example, taking the specific case of $\lambda=150 \mathrm{~m}^{-1}$, we find $\beta_{d} \simeq 1.41 \mathrm{~m}^{-1}$ which corresponds to the deconfining temperature $T_{d} \simeq 0.71 \mathrm{~m}$; this result could be illustrated in a figure with the same pattern as that appearing in Fig. 2 for the case where only one spatial coordinate is compactified. Again, the relation (21) holds with
$T_{d}^{\min } \simeq 0.54 \mathrm{~m}$, the deconfining temperature when $\lambda=\lambda_{c}$, and $T_{d}^{\max } \simeq 0.87 \mathrm{~m}$ being the upper bound, achieved when $\lambda \rightarrow \infty$.

Note that $T_{d}^{\mathrm{min}}$, for the fully compactified model, is smaller than the value when only one spatial dimension is compactified. This is because, in the present case, the modulus of the absolute minimum of $\sum_{32}^{R}\left(L_{1}, L_{2}\right)$ (which occurs for $\left.L_{1}=L_{2}=L_{\max }\right)$ is larger than that of $\Sigma_{31}^{R}(L)$, leading to a smaller critical value $\lambda_{c}$ when both spatial coordinates are compactified. On the other hand, $T_{d}^{\max }$ is the same for both cases of one or two spatial dimensions compactified. This is not a mere coincidence but reflects the fact that, in the extremely strong-coupling regime, the deconfining temperature is independent of the number of compactified spatial dimensions.

\section{CONCLUDING REMARKS}

We have analyzed the $N$-component 3-D massive GrossNeveu model with compactified spatial dimensions, both at zero and finite temperatures. The large- $N$ effective coupling constant $g$, for $T=0$, shows a kind of asymptotic freedom behavior, vanishing when the compactification length tends to zero, irrespective the value of the fixed coupling constant $\lambda$. In the strong coupling regime, where the fixed coupling constant is greater than some critical value, starting from small compactification lengths and increasing the size of the system, a divergence of the renormalized effective coupling constant appears at a given length, $L_{c}(\lambda)$, signaling that the system gets spatially confined. When the temperature is raised, a deconfining transition occurs at a temperature $T_{d}(\lambda)$, as the minimum of the inverse of the renormalized effective coupling constant reaches zero.

It should be emphasized that these results are intrinsic of the model and do not emerge from any adjustment. The limit values of $L_{c}(\lambda)$ and $T_{d}(\lambda)$ depend only on the fermion mass. Thus, to get an estimate of these values we have to fix the parameter $m$. To do so, we consider the Gross-Neveu model as an effective theory for the strong interaction (in which the gluon propagators have been shrunk, similarly to the Fermi treatment of the weak force) and take $m$ to be the constituent quark mass, $m \approx 350 \mathrm{MeV} \simeq 1.75 \mathrm{fm}^{-1}$ [7]. With such a choice, for the model with both spatial coordinates compactified, we find $0.74 \mathrm{fm}<L_{c}(\lambda)<1.20 \mathrm{fm}$ and, correspondingly, $305 \mathrm{MeV}>T_{d}(\lambda)>189 \mathrm{MeV}$. These values should be compared with the experimentally measured proton charge diameter $(\approx 1.74 \mathrm{fm})$ [8] and the estimated deconfining temperature $(\approx 200 \mathrm{MeV})$ for hadronic matter [9]. A detailed analysis of such a comparison, for arbitrary dimensions and in particular for $D=4$, will be presented elsewhere.

This work was partially supported by $\mathrm{CNPq}$ (Brazil) and NSERC (Canada). 
[1] D. J. Gross and A. Neveu, Phys. Rev. D 10, 3235 (1974).

[2] A. P. C. Malbouisson, J. M. C. Malbouisson, A. E. Santana, and J. C. Silva, Phys. Lett. B 583, 373 (2004).

[3] F. C. Khanna, A. P. C. Malbouisson, J. M. C. Malbouisson, H. Queiroz, T. M. Rocha-Filho, A. E. Santana, and J. C. Silva, Phys. Lett. B 624, 316 (2005).

[4] A. P. C. Malbouisson, J. M. C. Malbouisson, and A. E. Santana, Nucl. Phys. B 631, 83 (2002).
[5] A. Elizalde and E. Romeo, J. Math. Phys. 30, 1133 (1989).

[6] K. Kirsten, J. Math. Phys. 35, 459 (1994).

[7] Particle Data Group, Phys. Lett. B 592, 1 (2004); see page 475.

[8] S. G. Karshenboim, Can. J. Phys. 77, 241 (1999).

[9] A. Smilga, Lectures on QCD (World Scientific, Singapore, 2001) p. 279. 